\title{
Equivalência semântica da versão em português da Post-Traumatic Stress Disorder Checklist - Civilian Version (PCL-C) para rastreamento do transtorno de estresse pós-traumático
}

\author{
William Berger* \\ Mauro Vitor Mendlowicz** \\ Wanderson F. Souza*** \\ Ivan Figueira****
}

\section{INTRODUÇÃO}

O transtorno do estresse pós-traumático (TEPT) passou a ser reconhecido a partir da terceira edição do Manual diagnóstico e estatístico para transtornos mentais da Associação Psiquiátrica Americana (DSM-III), de $1980^{1}$. É caracterizado por uma reação de medo intenso, impotência ou horror quando um indivíduo vivencia, testemunha ou é confrontado com um ou mais eventos que envolvam morte, ferimento grave ou ameaça à integridade física, própria ou de outros (critério A para diagnóstico). A DSM-IV considera, ainda, o comunicado de um diagnóstico de doença grave, em que exista

Estudo realizado no Instituto de Psiquiatria da UFRJ, Departamento de Psiquiatria e Saúde Mental.

* Médico psiquiatra, aluno do estágio probatório do curso de Mestrado do Instituto de Psiquiatria da UFRJ (IPUB/UFRJ).

** Professor adjunto do Departamento de Psiquiatria e Saúde Mental da Universidade Federal Fluminense (UFF).

*** Estudante de Psicologia da Universidade Federal do Rio de Janeiro (UFRJ).

**** Professor adjunto da Faculdade de Medicina da Universidade Federal do Rio de Janeiro (UFRJ). risco de vida, como um estressor capaz de desencadear o TEPT ${ }^{2-4}$. Seus sintomas devem causar sofrimento clinicamente significativo, prejuízo social ou em outras áreas importantes do funcionamento, iniciar nos primeiros 6 meses após o evento (embora não obrigatoriamente, uma vez que a CID-10 considera a existência de TEPT tardio ${ }^{5}$ ) e ter duração superior a 1 mês. Apresenta três grupos de sintomas: revivescências (critério B: recordações aflitivas; pensamentos recorrentes; sonhos; flashbacks; etc.), comportamento evitativo/entorpecimento emocional (critério C: evitar pessoas, pensamentos, atividades ou lugares que lembrem o evento traumático; lapsos de memória; etc.) e hiperexcitabilidade (critério D: hipervigilância; insônia; resposta de sobressalto; etc. ${ }^{2,5-7}$ ).

Indivíduos portadores de TEPT apresentam pior qualidade de $v^{2} a^{3,8}$, maior número de detenções e problemas legais ${ }^{9}$, e utilizam com maior freqüência diversos serviços de saúde quando comparados a indivíduos sem o transtorno ${ }^{10,11}$. Tais fatos sugerem que o TEPT não afeta somente os 
indivíduos acometidos, mas também toda a sociedade, aumentando os gastos financeiros do Estado em vários setores, como saúde e segurança. Considerando ainda o aumento da violência urbana em nosso meio, torna-se clara a necessidade de novos estudos sobre este transtorno e, conseqüentemente, novos instrumentos de pesquisa adaptados à nossa língua, que sejam de fácil preenchimento e que possam ser utilizados em indivíduos de diversos níveis educacionais.

O TEPT atinge 2 a $5 \%$ da população geral em alguns países ${ }^{11}$, e dados sugerem que esta prevalência possa ser ainda maior no Brasil, dada a elevada prevalência de eventos traumáticos em nosso país. Por exemplo, temos a terceira maior taxa de mortalidade por arma de fogo entre jovens de 15 a 24 anos e somos um dos líderes mundiais em acidentes automobilísticos ${ }^{12}$. Apesar disso e do importante impacto pessoal e social inerentes ao TEPT, muito poucos estudos foram realizados no Brasil $^{13}$. Não dispomos, em nosso meio, por exemplo, de um instrumento de rastreamento para este transtorno, adaptado para a língua portuguesa (levando em consideração as peculiaridades brasileiras) e estudado quanto às suas características psicométricas.

$O$ presente artigo pretende descrever o método utilizado para comprovar a equivalência semântica da versão em português de uma escala auto-aplicável de rastreamento de TEPT, a Post-Traumatic Stress Disorder (PTSD) Checklist - Civilian Version (PCL-C), composta por 17 itens que avaliam os três grupos de sintomas descritos acima ${ }^{14}$.

\section{MÉTODOS}

\section{Instrumento original}

PCL-C ${ }^{14}$ : Instrumento desenvolvido em 1993 por Weathers, Litz, Huska e Keane, do National Center for PTSD (EUA), possui duas versões: a PCLC-M, especificamente desenvolvida para a avaliação das conseqüências de experiências militares, e a PCL-C, desenvolvida para a população civil, que avalia as conseqüências de diversos tipos de experiências traumáticas. Ambas as versões têm como base os critérios diagnósticos do DSM-III-R para o TEPT ${ }^{15}$. Para seu preenchimento, o examinado deve mensurar o quanto tem sido perturbado no último mês pelos sintomas descritos, utilizando uma escala de gravidade que varia original considera um escore maior ou igual a 3 (médio) em um dos 17 itens como um sintoma clinicamente significativo. Para o diagnóstico de TEPT, o indivíduo deve apresentar, além do critério $A$, um sintoma clinicamente significativo do critério $B$, três do $C$ e dois do $D$.

A versão original em inglês da PCL-C apresenta uma consistência interna (coeficiente alfa) de 0,90 para os critérios do grupo $B$, de 0,89 para os do $C$, de 0,91 para os do $D$, e de 0,96 para todos os 17 itens da escala (valores considerados muito altos) ${ }^{14}$. A correlação total de itens varia de 0,52 a 0,80 quando comparada à escala de Mississipi. Neste mesmo estudo, os autores demonstraram uma validade de convergência igual a 0,85 entre as duas escalas ${ }^{14}$. Ao comparar a PCL à Structured Clinical Interview for DSM-III-R (SCID), Weathers et al. encontraram uma sensibilidade igual a 0,82 , uma especificidade igual a 0,83 e um índice kappa de $0,64^{14}$. Foi demonstrada também uma forte correlação com outras escalas, como a Escala de Evento de Impacto e a Escala PK da MMPI- $2^{14}$. Blanchard et al. ${ }^{16}$ publicaram, em 1996, um estudo onde foram avaliadas as propriedades psicométricas da PCL-C em 40 vítimas de acidentes automobilísticos e crimes sexuais. O instrumento em questão foi comparado à Clinician Administered PTSD Scale (CAPS), utilizada como referência no estudo ${ }^{16}$. Este revelou que a PCL-C apresenta uma correlação igual a 0,929 e uma eficiência diagnóstica (medida estatística derivada de uma tabela de classificação $2 \times 2$, incluindo sensibilidade, especificidade, valores preditivos positivo e negativo, kappa, phi, odds ratio e relação de probabilidade) igual a 0,9 em relação à CAPS. Ao avaliar cada item da escala, os autores encontraram uma correlação variando entre 0,386 e 0,788 e uma eficiência diagnóstica maior ou igual a $0,7^{16}$.

\section{Obtenção da equivalência semântica}

A comprovação da equivalência semântica da versão em português do instrumento em questão foi feita segundo o modelo proposto por Reichenheim et al. ${ }^{17}$, que consiste em quatro etapas distintas: tradução, retradução, apreciação formal de equivalência e interlocução com a população-alvo.

Tradução: A partir do instrumento original em inglês, dois psiquiatras com grande 
experiência na área e fluentes na língua inglesa fizeram duas traduções independentes da PCL-C para o português, criando, assim, duas versões do mesmo instrumento (V1 e V2).

Retradução: Dois cidadãos americanos, ambos fluentes na língua portuguesa e com formação superior completa (não-relacionados à área de saúde) participaram deste estudo. Um deles recebeu a versão V1, e o outro, a V2. Estes americanos fizeram, então, a tradução de V1 e V2 do português para o inglês, criando, assim, as versões que chamaremos de V3 e V4. Tais traduções foram feitas independentemente e sem que cada tradutor tivesse conhecimento do autor de V1 ou V2.

Apreciação formal de equivalência e adaptação: Nesta etapa, as duas novas versões em inglês (V3 e V4) foram comparadas à versão original da PCL-C, levando-se em consideração se as sentenças haviam sido reescritas com as mesmas palavras (em sua forma literal) ou se seu sentido original havia sido preservado (equivalência semântica). Considerando-se esses dois critérios e utilizando-se a Visual Analogue Scale (VAS), cada sentença de V3 e V4 recebeu uma nota variando de 0 a 100. Tanto a comparação quanto a graduação de cada item de V3 e V4 foram feitas por outros dois indivíduos ligados à área de saúde mental, ambos com experiência em utilização de diversas escalas psicométricas, sendo o primeiro um médico com residência completa em psiquiatria e o segundo um estudante de psicologia. Esses profissionais compararam, então, a equivalência semântica de cada sentença de V1 e V2 com a versão original da PCL-C, sendo cada uma graduada nas seguintes categorias: inalterada (IN), pouco alterada (PA), muito alterada (MA) e completamente alterada (CA).

Por exemplo, o item 1 da PCL-C, em sua versão original, é: "Repeated, disturbing memories, thoughts, or images of a stressful experience from the past?". O primeiro psiquiatra, ao fazer a tradução para o português, criou sua V1: "Memória, pensamentos e imagens repetitivos e perturbadores referentes a uma experiência estressante do passado?". Ao fazer a retradução, criou-se a V3 deste primeiro item: "Memories, thoughts and repetitive and disturbing images about a stressful experience in the past?". Esta V3 foi, então, comparada com o primeiro item da escala original, recebendo a nota 100 pela VAS e sendo graduada como "inalterada" (IN). Porém, ao se repetir o mesmo processo com V2 e V4, o item não manteve seu significado com a mesma precisão, recebendo, assim, a nota 60 pela VAS e o conceito "pouco alterada" (PA). Este processo foi repetido em todos os 17 itens da PCL-C. De acordo com a graduação obtida em cada item analisado, foi escolhido o melhor item de cada uma das versões em português ( V1 e V2) para a composição de uma versão final nesta língua. Foi permitido que, em alguns itens, os autores selecionassem pequenos trechos de V1 e V2 e os fundissem, criando, assim, um item com as melhores partes de cada tradução. No item 12, permitiu-se, ainda, a criação de uma terceira versão, uma vez que nem V1 nem V2 foram consideradas satisfatórias para expressar o critério pesquisado na versão original em inglês. Em alguns casos, foram permitidas também pequenas correções gramaticais ou adaptações semânticas.

Interlocução com a população-alvo: Após a criação de uma versão final em português da PCL-C (anexo 1), na qual foi mantida inclusive a aparência original do instrumento criado por Weathers et al., esta foi apresentada a 21 brasileiros, escolhidos por conveniência, de diversos níveis educacionais e sociais (11 indivíduos de nível básico, seis de nível médio e quatro de nível superior). Essas pessoas preencheram a escala e também lhes foi perguntado sobre a compreensão de cada item da PCL-C, assim como sobre a existência de alguma sugestão a respeito do instrumento.

A figura 1 mostra de maneira esquemática toda a metodologia aplicada neste estudo. 


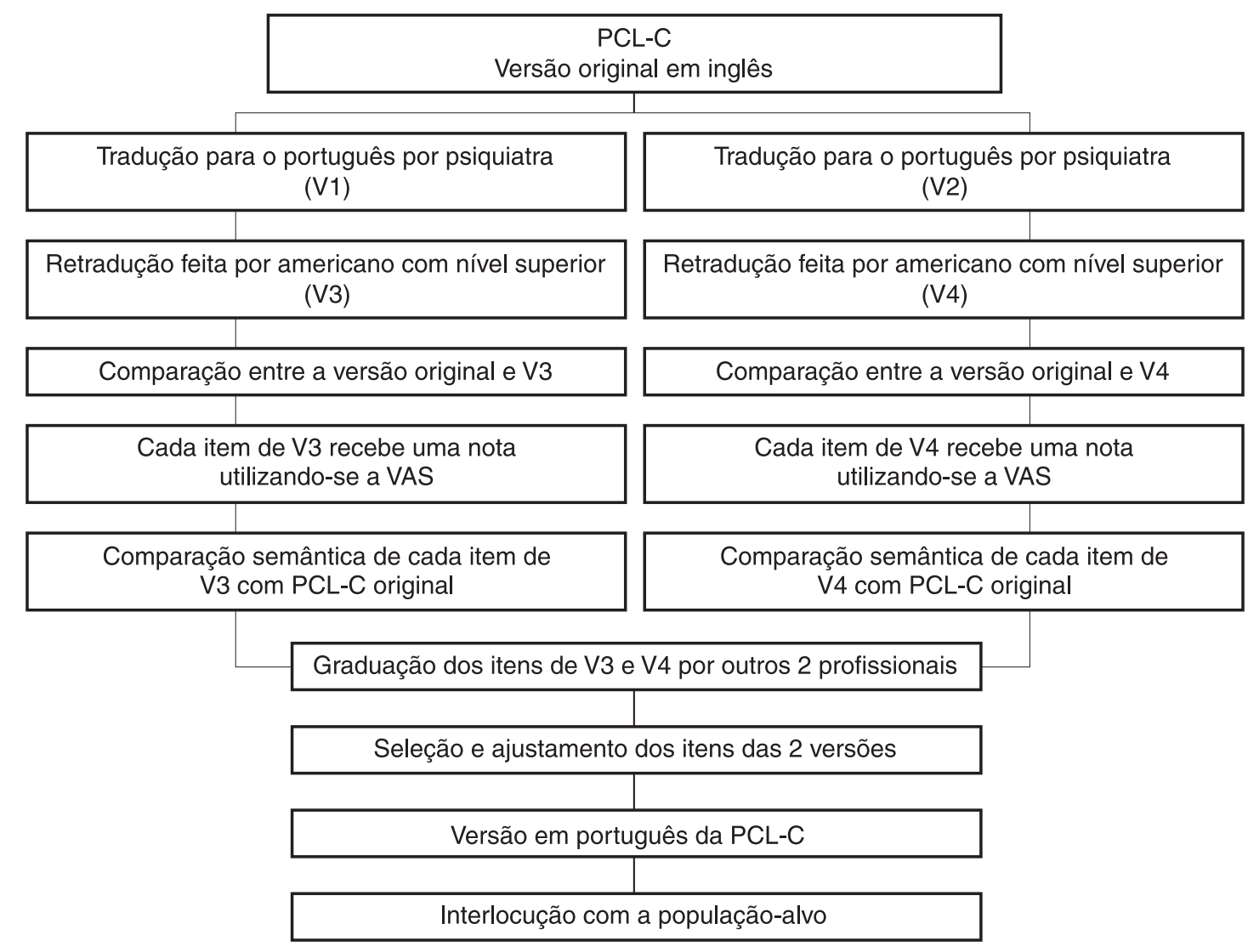

Figura 1 - Resumo esquemático da metodologia empregada para a comprovação da equivalência semântica da versão em português da PCL-C

\section{RESULTADOS}

Com o emprego da metodologia descrita acima, foi criada uma versão em português de um instrumento amplamente utilizado e validado em vários países do mundo. Foram respeitados os critérios diagnósticos para TEPT e até mesmo a aparência da versão original em inglês da PCL-C.

Ao apresentarmos a escala à população estudada, nenhum indivíduo apresentou dificuldades em seu preenchimento. Quando solicitamos que os indivíduos preenchessem a escala sem explicar o que esta objetivava medir, praticamente todos compreenderam o termo "situação de vida estressante", presente nas instruções iniciais, como dificuldades em seus empregos, e não como eventos traumáticos.

\section{DISCUSSÃO}

A PCL-C foi escolhida para este estudo por ser um instrumento para rastreamento de TEPT amplamente utilizado por diversos autores ${ }^{3,4,8-}$ 11,16,18-33 e com vários estudos de validação e determinação de suas propriedades psicométricas, em sua versão original em inglês e também em outros idiomas ${ }^{4,14,16,18,22,23,26,28,33,34}$. Possui a característica de ser auto-aplicável, ou seja, não necessita de um examinador externo, diferentemente de outros instrumentos existentes, como a Clinician Administered PTSD Scale for DSM-IV (CAPS-DX) ${ }^{35}$ e a SCID ${ }^{36}$. Dois outros instrumentos para rastreamento de TEPT amplamente utilizados em diversos estudos são a Escala de Trauma de Davidson (DST) ${ }^{37}$ e a Post-Traumatic Stress Diagnostic Scale $(\text { PDS })^{38}$. Entretanto, preferimos utilizar a PCL-C 
para a validação de face, pois esta tem sido utilizada em um espectro mais amplo de situações clínicas, como amostras de cuidado primário, pacientes com câncer e outras condições médicas em geral| ${ }^{3,4,8,11,18,21,24,25,39}$.

A PCL-C já foi estudada e aplicada a outras populações que não utilizam a língua inglesa como idioma principal (como em estudos conduzidos na Alemanha e em comunidades hispânicas dos EUA, em suas respectivas versões em alemão e espanhol) ${ }^{29,31,40}$, mas ainda não há nenhum estudo no Brasil que comprove a equivalência semântica de uma versão adaptada à nossa língua, assim como sua validade e confiabilidade.

O instrumento possui outras utilidades além do rastreamento do TEPT. Por exemplo, já foi utilizado em pesquisas clínicas para a avaliação de respostas ao tratamento deste transtorno, uma vez que a gravidade dos sintomas estudados pode ser avaliada de maneira continuada. Para isso, basta mudar a descrição dos sintomas de "último mês" para "última semana" e reaplicar a escala semanalmente, por exemplo ${ }^{14,20}$. A escala também já foi utilizada em diversos países com o objetivo de determinar a prevalência de TEPT após o diagnóstico de patologias graves, tratamentos muito agressivos (como quimioterapia e radioterapia, por exemplo) e até mesmo para comparar a evolução e o prognóstico de pacientes com e sem TEPT portadores desses diagnósticos ${ }^{3,4,8,18,21,24,25}$.

Uma limitação do presente estudo é o fato da escala ter sido apresentada a um número relativamente reduzido de pessoas $(n=21)$, produzindo, assim, um possível viés de espectro. A interlocução com a população-alvo mostrou que uma parte considerável da amostra estudada entendeu o termo "estresse" como problemas relacionados ao trabalho e rotinas de vida cansativas (emocional e fisicamente). Assim, imagina-se que a adição de uma pequena introdução que contextualize o que está sendo estudado, como a presente na Structured Clinical Interview for DSM-IV (SCID) ${ }^{36}$, aumente a sensibilidade e a especificidade (já elevadas) do instrumento original. Tal modificação, no entanto, alteraria as propriedades psicométricas do instrumento, não sendo assim realizada. Uma possível solução para este problema seria substituir o termo "situações de vida estressantes", presente no primeiro item das instruções do instrumento, por "situações de vida traumatizantes".

Estudos adicionais com a PCL-C devem ser empreendidos, uma vez que a comprovação da equivalência semântica ou a validação de face de uma escala é apenas uma das etapas para a validação de um instrumento em qualquer língua. Para um instrumento ser adequado para uso em pesquisa, este deve apresentar uma boa confiabilidade (capacidade do instrumento chegar ao mesmo diagnóstico em determinado paciente ou grupo diagnóstico quando aspectos da avaliação são alterados, como entrevistador ou momento da aplicação do instrumento) e validade (captar, identificar ou medir aquilo a que se propõe $)^{41}$. Deve-se considerar, ainda, a sensibilidade (capacidade de detecção de indivíduos portadores da patologia em questão) e a especificidade (capacidade de exclusão de indivíduos que não possuem a patologia estudada). Tais fatores ainda não foram determinados na aplicação da versão em português da PCL-C a brasileiros. A fim de se conhecer essas propriedades psicométricas da escala em questão adaptada à língua portuguesa, os autores deste artigo estão desenvolvendo outros estudos com uma amostra muito maior que a aqui citada.

\section{CONCLUSÕES}

Apesar de sua equivalência semântica ter sido constatada, estudos adicionais com a versão em português da PCL-C, já em andamento, deverão avaliar as características psicométricas deste instrumento na população brasileira. Por exemplo, é necessário determinar a eficiência diagnóstica deste instrumento tendo o SCID como padrão ouro, avaliar a confiabilidade teste-reteste e empregar a metodologia da curva ROC. Só assim tal instrumento terá passado por todas as etapas para sua validação comprovada para a população brasileira e poderá ser amplamente utilizado em nosso país.

Assim, com a criação da versão adaptada da PCL-C à população brasileira, cuja primeira etapa (validação de face) foi iniciada neste estudo, teremos um instrumento capaz de ajudar no diagnóstico e acompanhamento da evolução de um transtorno pouco estudado e que, provavelmente, causa extremo sofrimento a inúmeros brasileiros, o transtorno do estresse pós-traumático.

\section{Agradecimentos}

A Penélope Alves Breglia, por traduzir o resumo deste artigo para o espanhol. 


\section{REFERÊNCIAS BIBLIOGRÁFICAS}

1. American Psychiatry Association. Diagnostic and statistical manual for mental disorders. 3rd ed. Washington: American Psychiatric Press; 1980.

2. American Psychiatric Association. Diagnostic and statistical manual of mental disorders. 4th ed. Washington: American Psychiatric Press; 1994.

3. Cordova MJ, Andrykowski MA, Kenady DE, et al Frequency and correlates of posttraumatic-stress disorder-like symptoms after treatment for breast cancer. $\mathrm{J}$ Consult Clin Psychol 1995;63:981-6.

4. Smith MY, Redd W, DuHamel K, et al. Validation of the PTSD Checklist-Civilian Version in survivors of bone marrow transplantation. J Trauma Stress 1999;12:48599

5. World Health Organization. Classificação de transtornos mentais e de comportamento da CID-10. Porto Alegre: Artmed; 1993.

6. Kaplan HI, Sadock BJ. Comprehensive textbook of psychiatry. 7th ed. New York: Lippincott Williams \& Wilkins; 2000.

7. Yehuda R. Post-traumatic stress disorder. N Engl J Med 2002;346:108-13.

8. Smith MY, Egert J, Winkel G, et al. The impact of PTSD on pain experience in persons with HIV/AIDS. Pain 2002;98:9-17.

9. Saxon AJ, Davis TM, Sloan KL, et al. Trauma, symptoms of posttraumatic stress disorder, and associated problems among incarcerated veterans. Psychiatr Serv 2001;52:959-64

10. Rosenberg HJ, Rosenberg SD, Wolford GL, et al. The relationship between trauma, PTSD, and medica utilization in three high risk medical populations. Int $J$ Psychiatry Med 2000;30:247-59.

11. Stein MB, McQuaid JR, Pedrelli P, et al. Posttraumatic stress disorder in the primary care medical setting. Gen Hosp Psychiatry 2000;22:261-9.

12. Waiselfisz JJ. Mapa da Violência III. UNESCO/Instituto Ayrton Senna/Ministério da Justiça/SEDH; 2002.

13. Maurat A, Figueira I. Tratamento farmacológico do transtorno do estresse pós-traumático. Rev Psiquiatr Clin $2001 ; 28: 191-6$.

14. Weathers FW, Litz BT, Herman D, Huska JA, Keane TM. The PTSD Checklist (PCL): reliability, validity, and diagnostic utility. Paper present at the Annual Meeting of International Society for Traumatic Stress Studies, San Antonio, TX, October 1993.

15. American Psychiatric Association. Diagnostic and statistical manual of mental disorders. 3rd ed, revised. Washington: American Psychiatric Press; 1987.

16. Blanchard EB, Jones Alexander J, Buckley TC, et al. Psychometric properties of the PTSD checklist (PCL). Behav Res Ther 1996;34:669-73.

17. Reichenheim ME, Moraes $\mathrm{CL}$, Hasselmann $\mathrm{MH}$ Equivalência semântica da versão em português do instrumento Abuse Assessment Screen para rastrear a violência contra a mulher grávida. Rev Saude Publica 2000;34:610-6.

18. Andrykowski MA, Cordova MJ, Studts JL, et al. Posttraumatic stress disorder after treatment for breast cancer: prevalence of diagnosis and use of the PTSD Checklist-Civilian Version (PCL-C) as a screening instrument. J Consult Clin Psychol 1998;66:586-90.

19. Asmundson GJ, Frombach I, McQuaid J, et al. Dimensionality of posttraumatic stress symptoms: a confirmatory factor analysis of DSM-IV symptom clusters and other symptom models. Behav Res Ther 2000;38:203-14.

20. Berlant J, van Kammen DP. Open-label topiramate as primary or adjunctive therapy in chronic civilian posttraumatic stress disorder: a preliminary report. J Clin Psychiatry 2002;63:15-20

21. Cordova MJ, Studts JL, Hann DM, et al. Symptom structure of PTSD following breast cancer. J Trauma Stress 2000;13:301-19.

22. Dobie DJ, Kivlahan DR, Maynard C, et al. Screening for post-traumatic stress disorder in female Veteran's Affairs patients: validation of the PTSD checklist. Gen Hosp Psychiatry 2002;24:367-74.

23. Forbes D, Creamer M, Biddle D. The validity of the PTSD checklist as a measure of symptomatic change in combatrelated PTSD. Behav Res Ther 2001;39:977-86.

24. Mager WM, Andrykowski MA. Communication in the cancer 'bad news' consultation: patient perceptions and psychological adjustment. Psychooncology 2002;11:3546.

25. Manne SL, Du HK, Gallelli K, et al. Posttraumatic stress disorder among mothers of pediatric cancer survivors: diagnosis, comorbidity, and utility of the PTSD checklist as a screening instrument. J Pediatr Psychol 1998;23:357-66.

26. Mueser KT, Rosenberg SD, Fox L, et al. Psychometric evaluation of trauma and posttraumatic stress disorder assessments in persons with severe mental illness. Psychol Assess 2001;13:110-7.

27. Schlenger WE, Caddell JM, Ebert L, et al. Psychological reactions to terrorist attacks - findings from the national study of Americans' reactions to September 11. JAMA 2002;288:581-8.

28. Smith MY, DuHamel K, Vickberg SJ, et al. Reliability and validity of the post-traumatic stress disorder checklistcivilian (PCL-C). Psychosom Med 1997;59:85.

29. Teegen F, Muller J. [Trauma exposure and post-traumatic stress disorder in intensive care unit personnel]. Psychother Psychosom Med Psychol 2000;50:384-90.

30. Teegen F, Grotwinkel M. Traumatic exposure and posttraumatic stress disorder of journalists. An internet-based study. Psychotherapeut 2001;46:169-75.

31. Teegen F, Schriefer J. Complex posttraumatic stress disorder - analysis of a diagnostic construct in the case of abused women. Z Klin Psychol Psychiatr Psychother 2002;50:219-33.

32. Teegen F, Schriefer J. Domestic violence. Posttraumatic stress disorder of battered women. Psychotherapeut 2002;47:90-7.

33. Walker EA, Newman E, Dobie DJ, et al. Validation of the PTSD checklist in an HMO sample of women. Gen Hosp Psychiatry 2002;24: 375-80.

34. Campbell KA, Rohlman DS, Storzbach D, et al. Testretest reliability of psychological and neurobehavioral tests self-administered by computer. Assessment 1999;6:21-32.

35. Blake DD, Weathers FW, Nagy LM, Kaloupek DG, Charney DS, Keane TM. Clinician-administered PTSD scale for DSM-IV (CAPS-DX). Current and lifetime diagnostic version. 1997.

36. Spitzer RL, Williams JBW, Gibbon M, First MB. Structured clinical interview for DSM-IV (SCID). 1989.

37. Davidson JRT, Book SW, Colket JT, Tupler LA, Roth S, David D, Hertzberg M, Mellman T, Beckham JC, Smith R, Davison RM, Katz R, Feldman M. Davidson Trauma Scale (DTS). Psychol Med 1997;27(1):153-60.

38. Foa E, Cashman L, Jaycox L, Perry K. The validation of a self-report measure of posttraumatic stress disorder: the Posttraumatic Diagnostic Scale. Psychol Assess 1997;9:445-51

39. Sampson MJ, Kinderman $P$, Watts $S$, et al. Psychopathology and autobiographical memory in stroke and non- stroke hospitalized patients. Int $\mathrm{J}$ Geriatr Psychiatry 2003;18:23-32. 
40. Orlando M, Marshall GN. Differential item functioning in a Spanish translation of the PTSD checklist: detection and evaluation of impact. Psychol Assess 2002;14:50-9.

41. Dalgalarrondo $P$, ed. Ordenação dos fenômenos em psicopatologia: princípios gerais do diagnóstico. In: Psicopatologia e semiologia dos transtornos mentais. Porto Alegre: Artmed; 2000. pp. 31-5.

\section{RESUMO}

Introdução: O transtorno do estresse póstraumático (TEPT) é bastante prevalente, atingindo de 2 a $5 \%$ da população em alguns países. Seus portadores apresentam pior qualidade de vida, maior número de detenções e problemas legais, e utilizam os serviços de saúde com maior freqüência quando comparados a indivíduos não acometidos. Apesar do crescimento da violência urbana no Brasil, ainda não dispomos de nenhum instrumento adaptado para nossa língua que seja capaz de rastrear o TEPT. Este artigo pretende traduzir, adaptar e avaliar a aplicabilidade da versão em português da PostTraumatic Stress Disorder Checklist - Civilian Version (PCL-C), uma escala auto-aplicável amplamente utilizada em todo o mundo para rastreamento do transtorno do estresse pós-traumático.

Métodos: A comprovação da equivalência semântica da versão em português da PCL-C foi feita através de quatro etapas: tradução; retradução; apreciação formal de equivalência e adaptação; $e$ interlocução com a população-alvo.

Resultados: Foi criada uma versão em português da PCL-C que manteve o significado semântico da versão original e mostrou-se de fácil compreensão e aplicabilidade.

Discussão: A PCL-C foi escolhida para este estudo por ser um instrumento amplamente utilizado em países de língua inglesa e por possuir outras utilidades além do rastreamento do TEPT. A interlocução com a população-alvo, feita com um número relativamente pequeno de indivíduos $(n=$ 21), mostrou que alguns participantes entenderam o termo "estresse" como uma rotina de vida cansativa.

Conclusão: Uma vez obtida a equivalência semântica da versão em português da PCL-C, estudos posteriores deverão determinar suas características psicométricas para a população brasileira.

Descritores: Transtorno do estresse pós-traumático, TEPT, PCL-C, instrumento de rastreamento, equivalência semântica.

\section{ABSTRACT}

Background: The prevalence of post-traumatic stress disorder (PTSD) is high - it affects $2-5 \%$ of the population in some countries. These patients present worse quality of life, a higher number of detentions and legal problems, and use healthcare services more frequently than individuals without the disorder.
Despite the growth of urban violence in Brazil, no instrument is currently available, in the Portuguese language, aimed at screening TEPT. The objective of this study was to translate, adapt and assess the applicability of the Portuguese version of the PTSD Checklist - Civilian Version (PCL-C), a selfadministered scale for PTSD screening widely used in several countries.

Methods: Semantic equivalence between the Portuguese version and the original PCL-C was assessed through four stages: translation; back translation; formal equivalence comparison and adaptation; and interlocution with the target population.

Results: A Portuguese version for PCL-C was created preserving the meaning of the original version and showed to be easily applicable.

Discussion: The PCL-C was chosen in this study because it is a widely used instrument in Englishspeaking countries and has others utilities than the screening of PTSD. The interlocution with the target population, carried out with a relatively low number of patients $(n=21)$, showed that some participants understood the term "stress" as referring to a stressful lifestyle.

Conclusions: Now that semantic equivalence of the Portuguese version of PCL-C was established, further studies are necessary in order to determine its psychometric properties for the Brazilian population.

Keywords: Post-traumatic distress disorder, PTSD, $P C L-C$, screening tool, semantic equivalence.

Title: Semantic equivalence of the Portuguese version of the Post-Traumatic Stress Disorder Checklist Civilian Version (PCL-C) for the screening of posttraumatic stress disorder

\section{RESUMEN}

Introducción: El trastorno de estrés postraumático (TEPT) es bastante común, afectando del 2 al 5\% de la población en algunos países. Quienes lo padecen presentan una inferior calidad de vida, un mayor número de detenciones y problemas legales y recurren más frecuentemente a los servicios de salud que los individuos no afectados. A pesar del incremento de la violencia urbana en Brasil, todavía no disponemos de ningún instrumento adaptado a nuestro idioma que sea capaz de rastrear el TEPT. Este artículo pretende traducir, adaptar y evaluar la aplicabilidad de la versión en portugués de la PostTraumatic Stress Disorder Checklist - Civilian Version (PCL-C), una escala auto-aplicable extensamente utilizada en todo el mundo para el rastreo del Trastorno de Estrés Postraumático.

Métodos: Se comprobó la equivalencia semántica de la versión en portugués de la PCL-C a través de 4 etapas: traducción; re-traducción; apreciación formal de la equivalencia y adaptación; e interlocución con la población objetivo.

Resultados: Se creó una versión en Portugués 
de la PCL-C que mantuvo el significado semántico de la versión original y se presentó fácilmente comprensible y aplicable.

Debate: Se eligió la PCL-C para este estudio por ser un instrumento ampliamente utilizado en países de lengua inglesa y por tener otras utilidades más allá del rastreo del TEPT. La interlocución con la población objetivo, realizada con un número relativamente pequeño de individuos $(n=21)$, mostró que algunos participantes interpretaron el término "estrés" como una rutina fatigante de vida.

Conclusión: Una vez que se obtenga la equivalencia semántica de la versión en portugués de la PCL-C, estudios posteriores deberán determinar sus características psicométricas para la población brasileña.
Palabras clave: Trastorno de estrés postraumático, TEPT, PCL-C, instrumento de rastreo, equivalencia semántica.

Título: Equivalencia semántica de la versión en portugués de la Post-Traumatic Stress Disorder Checklist - Civilian Version (PCL-C) para el rastreo del trastorno de estrés pos-traumático

Endereço para correspondência:

William Berger

Rua Doutor Satamini, 90/202 - Tijuca

CEP 20270-230 - Rio de Janeiro - RJ

Fone: (21) 2234-2304/9643-6353

E-mail: wberger@globo.com

Copyright (C) Revista de Psiquiatria do Rio Grande do Sul - SPRS 


\section{ANEXO 1: PCL-C}

Instruções:

Abaixo, há uma lista de problemas e de queixas que as pessoas às vezes apresentam como uma reação a situações de vida estressantes.

Por favor, indique o quanto você foi incomodado por estes problemas durante o último mês.

Por favor, marque 1 para "nada", 2 para "um pouco", 3 para "médio", 4 para "bastante" e 5 para "muito".

\begin{tabular}{|l|c|c|c|c|c|}
\hline & Nada & Um pouco & Médio & Bastante & Muito \\
\hline $\begin{array}{l}\text { 1. Memória, pensamentos e imagens repetitivos e perturbadores } \\
\text { referentes a uma experiência estressante do passado? }\end{array}$ & 1 & 2 & 3 & 4 & 5 \\
\hline $\begin{array}{l}\text { 2. Sonhos repetitivos e perturbadores referentes a uma experiência } \\
\text { estressante do passado? }\end{array}$ & 1 & 2 & 3 & 4 & 5 \\
\hline $\begin{array}{l}\text { 3. De repente, agir ou sentir como se uma experiência estressante } \\
\text { do passado estivesse acontecendo de novo (como se você a } \\
\text { estivesse revivendo)? }\end{array}$ & 1 & 2 & 3 & 4 & 5 \\
\hline $\begin{array}{l}\text { 4. Sentir-se muito chateado ou preocupado quando alguma coisa } \\
\text { lembra você de uma experiência estressante do passado? }\end{array}$ & 1 & 2 & 3 & 4 & 5 \\
\hline $\begin{array}{l}\text { 5. Sentir sintomas físicos (por exemplo, coração batendo forte, } \\
\text { dificuldade de respirar, suores) quando alguma coisa lembra você de } \\
\text { uma experiência estressante do passado? }\end{array}$ & 1 & 2 & 3 & 4 & 5 \\
\hline $\begin{array}{l}\text { 6. Evitar pensar ou falar sobre uma experiência estressante do } \\
\text { passado ou evitar ter sentimentos relacionados a esta experiência? }\end{array}$ & 1 & 2 & 3 & 4 & 5 \\
\hline $\begin{array}{l}\text { 7. Evitar atividades ou situações porque elas lembram uma } \\
\text { experiência estressante do passado? }\end{array}$ & 1 & 2 & 3 & 4 & 5 \\
\hline $\begin{array}{l}\text { 8. Dificuldades para lembrar-se de partes importantes de uma } \\
\text { experiência estressante do passado? }\end{array}$ & 1 & 2 & 3 & 4 & 5 \\
\hline $\begin{array}{l}\text { 9. Perda de interesse nas atividades de que você antes costumava } \\
\text { gostar? }\end{array}$ & 1 & 2 & 3 & 4 & 5 \\
\hline $\begin{array}{l}\text { 10. Sentir-se distante ou afastado das outras pessoas? } \\
\text { 11. Sentir-se emocionalmente entorpecido ou incapaz de ter } \\
\text { sentimentos amorosos pelas pessoas que Ihe são próximas? }\end{array}$ & 1 & 2 & 3 & 4 & 5 \\
\hline $\begin{array}{l}\text { 12. Sentir como se você não tivesse expectativas para o futuro? } \\
\text { 13. Ter problemas para pegar no sono ou para continuar dormindo? }\end{array}$ & 1 & 2 & 3 & 4 & 5 \\
\hline $\begin{array}{l}\text { 14. Sentir-se irritável ou ter explosões de raiva? } \\
\text { 16. Estar "superalerta", vigilante ou "em guarda"? tenso ou facilmente sobressaltado? }\end{array}$ & 1 & 2 & 3 & 4 & 5 \\
\hline 15. Ter dificuldades para se concentrar? & 1 & 2 & 3 & 4 & 5 \\
\hline $\begin{array}{l}\text { 16 } \\
\text { 17. }\end{array}$ & 2 & 3 & 4 & 5 \\
\hline
\end{tabular}

